\title{
Coherent Interaction of Light and Single Molecules in a Dielectric Nanoguide
}

\author{
Sanli Faez, ${ }^{1,2}$ Pierre Türschmann, ${ }^{1}$ Harald R. Haakh, ${ }^{1}$ Stephan Götzinger, ${ }^{3,1}$ and Vahid Sandoghdar ${ }^{1,3}$ \\ ${ }^{1}$ Max Planck Institute for the Science of Light (MPL), D-91058 Erlangen, Germany \\ ${ }^{2}$ Leiden Institute of Physics, P.O. Box 9504, 2300 RA Leiden, The Netherlands \\ ${ }^{3}$ Department of Physics, Friedrich Alexander University of Erlangen-Nürnberg, D-91058 Erlangen, Germany
}

(Received 9 July 2014; published 18 November 2014)

\begin{abstract}
Many of the currently pursued experiments in quantum optics would greatly benefit from a strong interaction between light and matter. Here, we present a simple new scheme for the efficient coupling of single molecules and photons. A glass capillary with a diameter of $600 \mathrm{~nm}$ filled with an organic crystal tightly guides the excitation light and provides a maximum spontaneous emission coupling factor $(\beta)$ of $18 \%$ for the dye molecules doped in the organic crystal. A combination of extinction, fluorescence excitation, and resonance fluorescence spectroscopy with microscopy provides high-resolution spatiospectral access to a very large number of single molecules in a linear geometry. We discuss strategies for exploring a range of quantum-optical phenomena, including polaritonic interactions in a mesoscopic ensemble of molecules mediated by a single mode of propagating photons.
\end{abstract}

PACS numbers: 42.50.- $\mathrm{p}, 33.80 .-\mathrm{b}, 42.50 . \mathrm{Nn}, 42.81 . \mathrm{Qb}$

The interaction of light and matter has played a prominent role in quantum physics and continues to kindle new areas of research. One of the exciting emerging activities is to extend strongly correlated many-body interactions known for electrons in a solid to the context of combined atomphoton states, also referred to as polaritons. Examples of proposed phenomena in such photonic condensed matter include the Luttinger liquid of photons [1], quantum phase transitions in light [2], a quantum-optical Josephson interferometer [3], and the Tonks-Girardeau gas of photons [4,5]. The success of these and many other recent ideas [6,7], however, will rely on the efficiency of the coupling between material and light particles. The most common route towards enhancing this interaction has been to trap and circulate photons in a high-finesse microcavity [2,8-10], but it has been pointed out that it is even possible for propagating photons to reach a substantial coupling with individual quantum emitters directly [11]. This idea has recently been experimentally demonstrated in a number of systems, including molecules, quantum dots, atoms, and ions [12-17].

An intuitive explanation for an efficient cavity-free interaction is that the extinction cross section of a two-level atom given by $\sigma_{0}=3 \lambda^{2} / 2 \pi$ can become comparable with the area of the excitation light, e.g., confined by a subwavelength aperture [12] or tight focusing [13-17]. The key concept for maximizing the coupling efficiency is, however, spatial mode matching [11]. In this picture, a tightly confined beam is a good choice because its large dipolar content is well adapted to the dipolar emission pattern of an atom in free space [18]. This point of view invokes an alternative strategy for optimizing light-matter coupling efficiency: one can modify the spatial mode of the atomic radiation to match the profile of the incident light beam, e.g., by employing parabolic [19] or planar [20] antennas. Similarly, it has been shown that a dielectric fiber with a subwavelength diameter can also channel the emission of an atom into its fundamental guiding mode $[21,22]$ and thus can act as an antenna. In what follows,
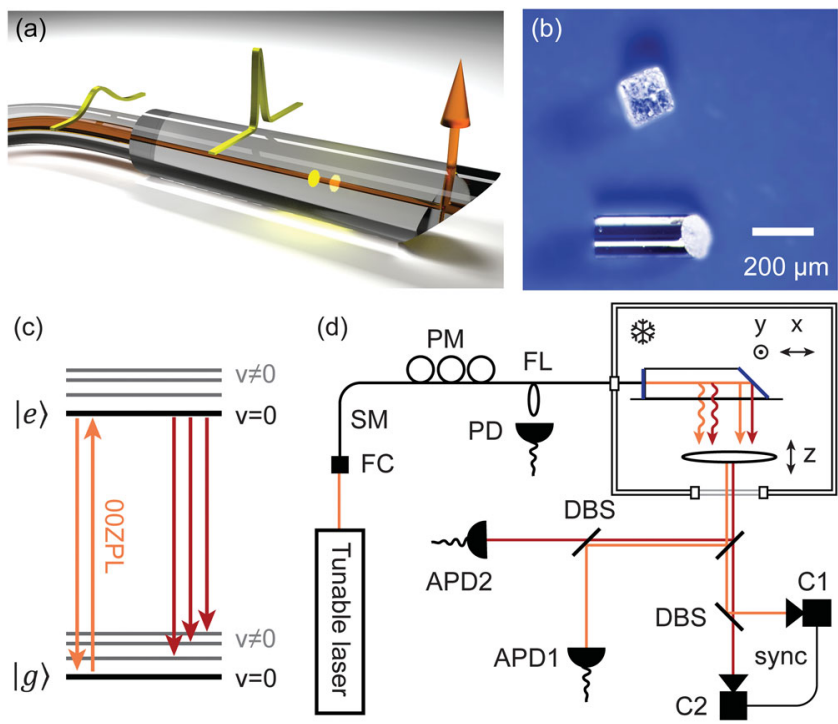

FIG. 1 (color). (a) Schematics of a filled capillary butt coupled to a single-mode optical fiber. (b) A microscopic image of an empty capillary next to a grain of table salt. (c) A simplified Jablonski diagram of a DBT molecule. (d) Sketch of the experimental arrangement. A cw Ti:sapphire laser is coupled to a single-mode fiber that enters a helium bath cryostat, ending with the nanocapillary. An aspheric lens provides optical access to the sample from a side window. Synchronized cameras C1 and C2 and avalanche photodiodes APD1 and APD2 are used for detection. PD: photodiode; SM: single-mode fiber; DBS: dichromatic beam splitter; PM: fiber polarization management; FC: fiber coupler; FL: fiber leakage for intensity stabilization. 
we generally refer to such a subwavelength waveguide antenna as a "nanoguide."

Interestingly, discussions of nanoguides have emerged somewhat in parallel to the tight focusing approach in different theoretical treatments [21-24] and experimental studies of glass nanofibers [25-27], plasmonic nanowires [28], photonic crystal waveguides [29,30], and even microwave transmission lines [31]. Moreover, scientists have investigated nonlinear optical effects in hollow-core fibers [32,33], although the mode in these structures is considerably less confined than in a nanoguide. In this Letter we present a new system, where molecules are coupled to the optical mode of a nanoguide with a core made of an organic crystal surrounded by a thick glass cladding. This arrangement lays the groundwork for controlled experiments on a one-dimensional ensemble of quantum emitters coupled via a photonic channel.

Figure 1(a) sketches the heart of our experimental setup. A fused silica capillary (refractive index $n_{2}=1.45$ ) with an inner diameter of $600 \mathrm{~nm}$ and an outer diameter of $160 \mu \mathrm{m}$ was cut to a length of about $400 \mu \mathrm{m}$ with one cleaved end and a second polished facet at $45^{\circ}$. Figure 1(b) shows a microscopic image of an empty capillary next to a grain of table salt. The flat and angled ends of the nanocapillary were coated with 65 and $45 \mathrm{~nm}$ layers of aluminum, respectively. Once this central piece was fabricated, we heated it in the presence of a small amount of naphthalene $\left(n_{1}=1.59\right)$ doped with dibenzoterrylene (DBT) molecules as quantum emitters at a concentration of $10^{-7} \mathrm{M}$. After capillary forces filled the core, we cooled the sample and removed the residual naphthalene from the surroundings. Here, it is important that one obtains a homogeneous core to maintain a guiding mode and reduce scattering losses from grain boundaries. Since the vapor pressure of naphthalene is high at ambient conditions, the first few micrometers usually turn up empty. Therefore, we filled this gap with ultraviolet curing glue (OG-142, Epotek). The resulting nanoguide only supports its fundamental mode.

DBT molecules in the nanoguide can be spectrally isolated because, as is common in solid-state media, the resonance frequencies of the individual molecules are distributed over an inhomogeneous spectrum [34]. The energy level scheme of DBT is illustrated in Fig. 1(c). The transition of interest for this work is labeled 00ZPL, which involves the zero-phonon line (ZPL) of the transition between the ground vibrational levels $(\mathrm{v}=0)$ of the electronic ground $(|g\rangle)$ and excited $(|e\rangle)$ states. The 00ZPL of DBT is known to have a lifetimelimited linewidth of $30 \mathrm{MHz}$ in naphthalene [35]. However, the Frank-Condon and Debye-Waller factors of DBT reduce the resonant fluorescence contribution of 00ZPL by an overall branching ratio of $\alpha \approx 0.3$. To gain access to the narrow resonances of individual molecules, we used a tunable Ti:sapphire ring laser with a linewidth of $100 \mathrm{kHz}$ and performed the experiment at $T \lesssim 1.5 \mathrm{~K}$ in a helium bath cryostat [see Fig. 1(d)].
To couple the excitation laser light to the nanoguide, we fed the laser light into the cryostat through a single-mode optical fiber and butt coupled it to the cleaved end of the capillary [see Fig. 1(a)]. The aluminium coating at this end suppresses any scattering into the capillary cladding, which is caused by the mismatch between the mode areas of $14 \mu \mathrm{m}^{2}$ for the single-mode fiber and $0.3 \mu \mathrm{m}^{2}$ for the nanoguide. We covered the capillary by index-matched ultraviolet curing glue to achieve nearly distortion-free imaging of the nanoguide from the side and to fix the relative alignment of the capillary and the single-mode fiber on a cover glass. Nanopositioning systems mounted in the cryostat allowed us to position the nanoguide inside of the lens field of view of about $120 \mu \mathrm{m} \times 120 \mu \mathrm{m}$ and to adjust its focus [see Fig. 1(d)]. In this fashion, we could record either the emission from the side of the nanoguide or the transmission via the $45^{\circ}$ mirror at its end.

The polarization of the excitation light was adjusted by a paddle polarization controller. We sampled the intensity inside the single-mode fiber by inducing a small leakage [see Fig. 1(d)] and used this signal to stabilize the excitation intensity against variations caused by thermal and mechanical perturbations. The light collected via the asphere was then sent to two synchronized detectors, which could be either sensitive cameras or avalanche photodiodes (APDs). In each case, a dichromatic beam splitter separated the excitation laser light from the Stokes-shifted fluorescence.

Figure 2(a) presents a conventional fluorescence excitation spectrum recorded through the angled output of the capillary. Here the narrow-band laser frequency is scanned and the Stokes-shifted fluorescence [transitions from $|e, v=0\rangle$ to $|g, v \neq 0\rangle$; see Fig. 1(c)] is registered through spectral filtering. Figure 2(b) displays a transmission spectrum recorded from the same port at a later time. Extinction dips of a few percent are observed when the resonance of a single molecule coincides with the laser frequency. The extinguished power $\Delta P$ at each dip results from the interference of the incident light at power $P_{0}$ with the light that is coherently scattered by an emitter into that mode [11-17]. It follows that $\Delta P / P_{0}=$ $2 \beta-\beta^{2}$, where $\beta$ stands for the fraction of the dipolar emission into the nanoguide mode compared to its overall radiation [36-38]. Accounting for the internal branching ratio $\alpha$ of the molecule, one then arrives at $\Delta P / P_{0}=$ $2(\alpha \beta)-(\alpha \beta)^{2}$. In Fig. 2(c) we show an example of an extinction dip reaching $6.5 \%$ or, equivalently, $\beta \approx 11 \%$. To verify that this signal stems from a single molecule, we performed Hanbury-Brown-Twiss coincidence measurements on this resonance. The outcome presented in Fig. 2(d) confirms antibunched emission with a lifetime of $5.3 \mathrm{~ns}$, corresponding to the measured linewidth of $\gamma=30 \mathrm{MHz}$.

As shown in Fig. 2(b), the recorded extinction dips display a certain degree of variation and, in fact, we have observed dips as large as $9 \%$. We attribute this distribution to the radial position of the molecules and the orientation of their dipole moments. Considering that the volume of a cylindrical shell at a distance $r$ from the center grows 

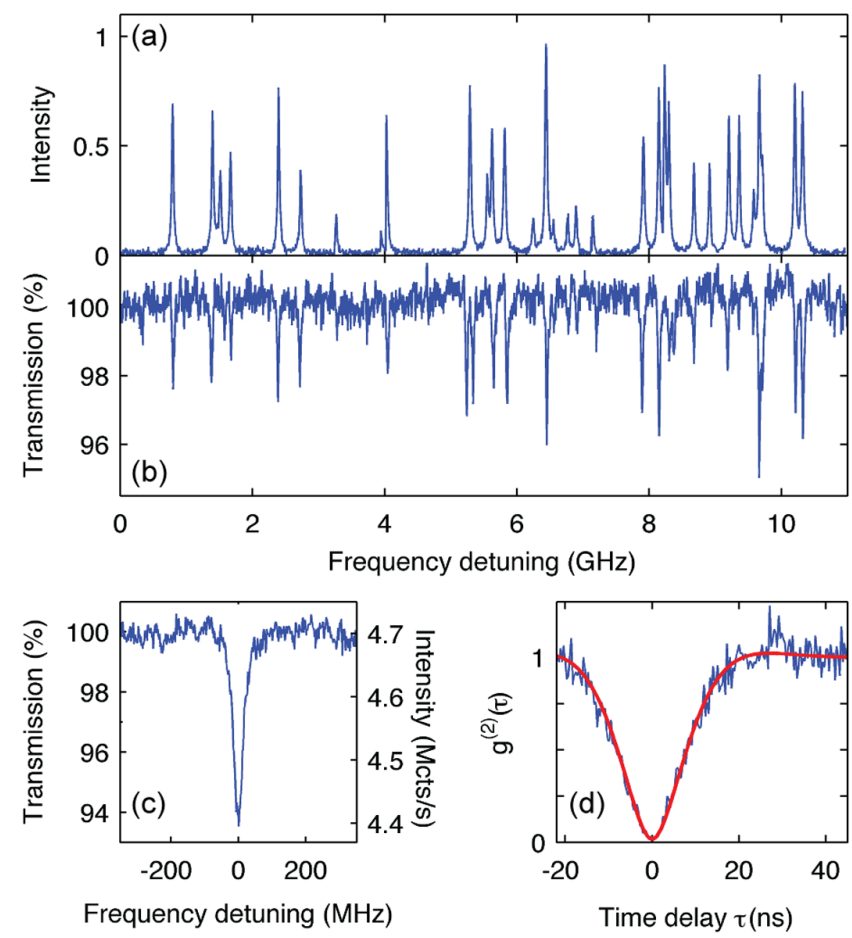

FIG. 2 (color online). (a) Stokes-shifted fluorescence spectrum recorded via the angled end of the nanoguide. (b) Extinction spectrum recorded directly in transmission, i.e., without using lock-in detection. This spectrum was recorded some tens of minutes after (a). While the great majority of the resonances are reproduced, in a few cases one notices slight frequency jumps. (c) A zoom into an extinction dip of $6.5 \%$ recorded from a single molecule. The excitation wavelength for the data in this figure was around $771 \mathrm{~nm}$. The right vertical axis shows the intensity of the excitation light in units of megacounts per second. (d) Second-order autocorrelation function of the photons emitted on the resonance of (c). Here, we detected the Stokes-shifted fluorescence from the side. The recorded value of $g^{(2)}(0)=0.01$ was limited by the APD dark counts.

linearly with $r$, a smaller fraction of the molecules experience the highest optical coupling.

The black curve in Fig. 3(a) plots the calculated values of $\beta$ for radially oriented dipole moments as a function of $r$. The expected range of $\beta=7 \%-18 \%$ translates to extinction dips of $4 \%-11 \%$ in our setup when considering $\alpha$. Finally, we emphasize that the inner radius of the capillary in our experiment was chosen to yield the highest coupling for the refractive indices of a naphthalene core and a glass cladding. The results of the calculations reported in Fig. 3(b) show the variation of $\beta$ and the mode area as a function of the capillary inner radius.

To present a thorough consideration of the radiative properties of a molecule coupled to the nanoguide, we also calculated the cavity quantum electrodynamic effect of the boundaries. The dashed blue curve in Fig. 3(a) indicates that the spontaneous emission rate of a radially oriented dipolar emitter changes by less than $20 \%$ across the nanoguide diameter. This level of variation is negligible in our work because the linewidths of dye molecules in
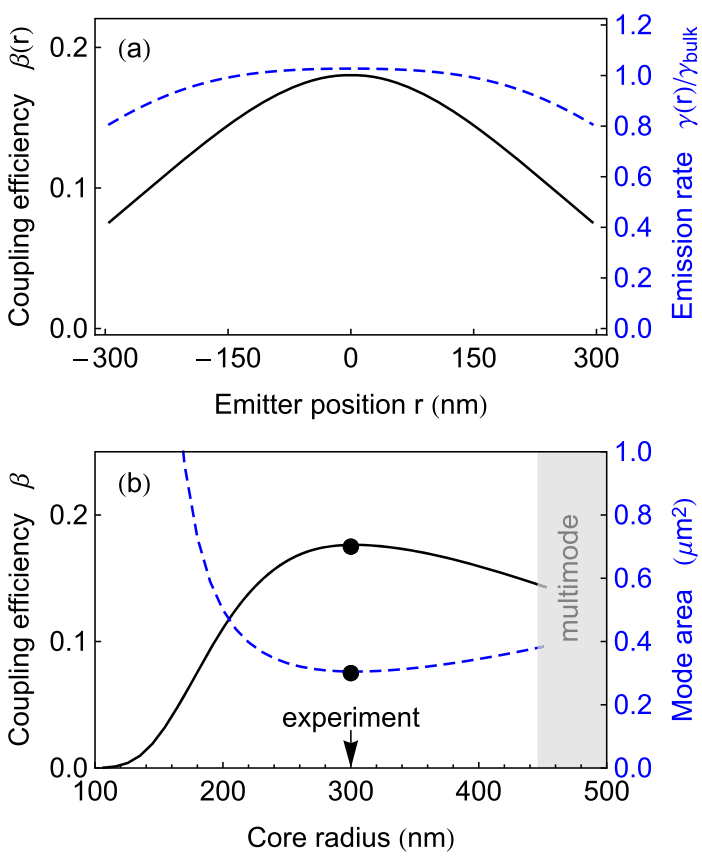

FIG. 3 (color online). (a) Nanoguide-emitter coupling efficiency $\beta$ (black solid curve, left axis) and the total decay rate (blue dashed curve, right axis) as a function of the emitter position inside a nanocapillary core of $600 \mathrm{~nm}$. (b) $\beta$ as a function of the core radius (black solid curve, left axis). The blue dashed trace plots the effective mode area (right axis). The dot indicates the optimal core radius $r=300 \mathrm{~nm}$, which was used in the experiment. The nanocapillary core was assumed to have a refractive index of $n_{1}=1.59$ surrounded by a large cladding with $n_{2}=1.45$. The shaded region in (b) marks the regime where the waveguide becomes multimode.

organic hosts commonly show a larger distribution [39]. In fact, we suspect that the crystallization process in the highly confined space of a nanocapillary might lead to additional mechanical stress and spectral inhomogeneity. We present a distribution of the measured linewidths in Fig. S2 of the Supplemental Material [38], but more detailed investigation of the homogeneous and inhomogeneous spectra in this novel geometry requires further studies.

Aside from achieving a high efficiency, our experimental arrangement provides access to the resonance fluorescence of molecules on a low background. Figure 4(a) displays an example of a single-molecule excitation spectrum obtained from the side of the nanoguide through a bandpass filter that transmits the excitation wavelength. This detection scheme offers a novel configuration for right-angle spectroscopy on molecules in a matrix. Although such measurements have been standard in gas-phase atomic and molecular physics [40], they are very challenging in the solid state due to strong background scattering. Figure 4(b) presents the spectrum of the same molecule as in Fig. 4(a) but detected through its Stokes-shifted fluorescence from the side. Monitoring the polarization of the detected fluorescence, we verified that the dipole moments of the molecules were generally aligned at an angle of about $80^{\circ}$ 

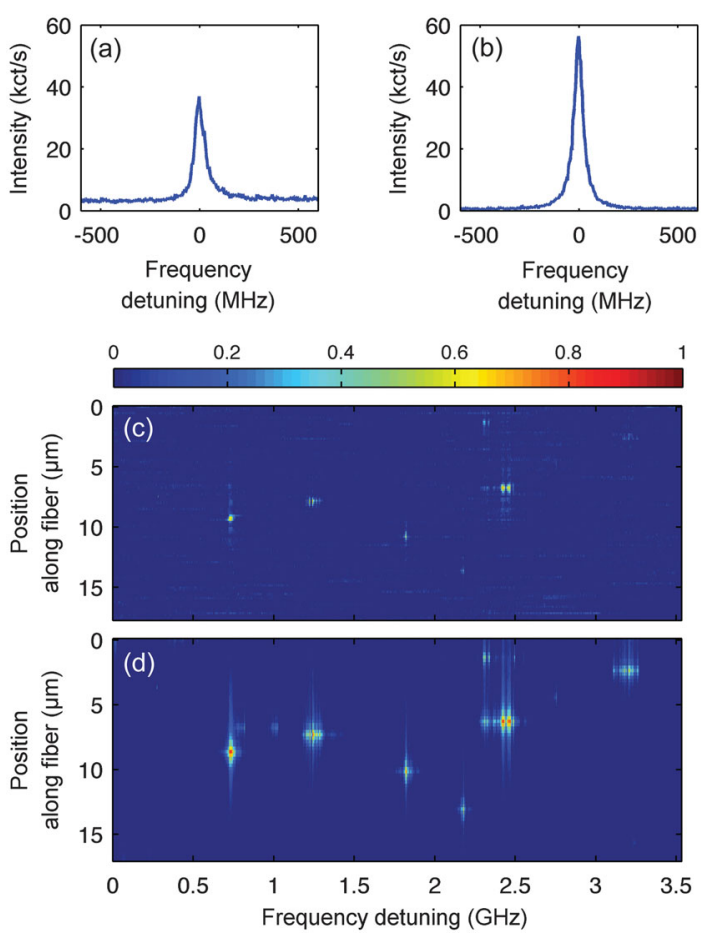

FIG. 4 (color). (a) A resonance fluorescence spectrum of a single DBT molecule recorded from the side. (b) The Stokesshifted fluorescence spectrum of the same molecule. (c) A section of the resonance fluorescence excitation spatiospectral map of the sample. (d) The same for the Stokes-shifted signal recorded simultaneously. The data in this figure were recorded at an excitation wavelength of around $757 \mathrm{~nm}$. The linear color scales represent the signal strength in arbitrary units.

relative to the nanoguide axis. However, rotation of the incident polarization within the nanoguide indicated that the radial dipole component did not have a fixed direction.

The side port of the nanoguide also brings about the important feature that all of the molecules across the thin capillary core can be imaged at the same time since they lie within the depth of focus of the aspheric lens. Figures 4(c) and 4(d) show spatiospectral maps of the resonance and Stokes-shifted fluorescence along more than $15 \mu \mathrm{m}$ of the nanoguide and within a spectral range of about $3 \mathrm{GHz}$. Considering that one can localize the center of the dipole moment of each molecule with angstrom precision through the analysis of its image point-spread function [41], the data in Fig. 4 illustrate the ability to address each molecule through high-resolution spectral and spatial information.

A noteworthy aspect of our system is that all molecules in the nanoguide lie well within a coherence length $l_{c}$ of each other, where $l_{c}=\tau c / n_{1} \sim 1 \mathrm{~m}$. Consequently, an efficient coupling of the molecular emission to the nanoguide mode $(\beta \sim 1)$ would lead to a novel long-range coherent coupling [42] analogous to what is known from near-field dipole-dipole interactions [43]. The first steps towards the realization of such a photonic coupling have been taken using two molecules in free space [44] and two superconducting qubits along a waveguide [45]. The

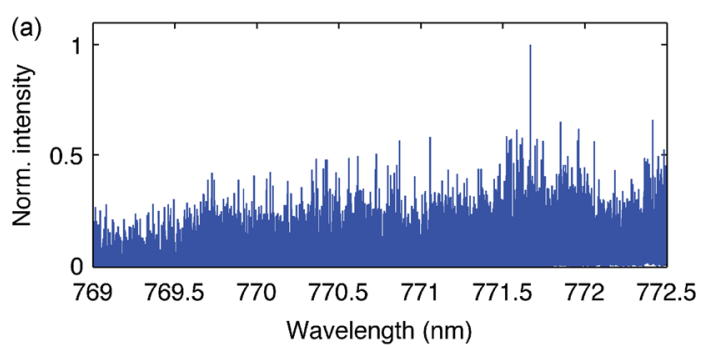

(b)

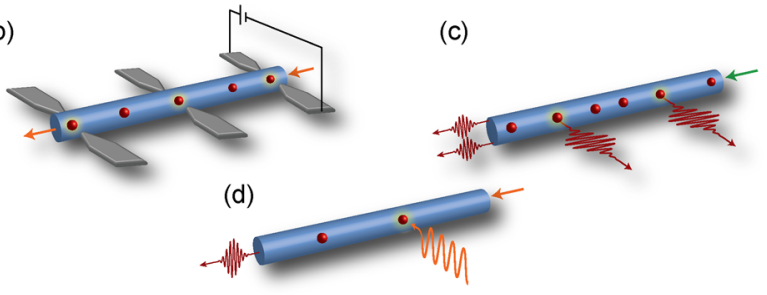

FIG. 5 (color online). (a) Stokes-shifted excitation spectrum recorded in transmission. The inhomogeneous distribution of DBT lines in our system is considerably larger than that observed in sublimated naphthalene crystals. Furthermore, the current site in the region of $770 \mathrm{~nm}$ has not been reported previously. (b) Schematics of micro- or nanoelectrodes for controlling the resonance frequencies of the molecules along the nanoguide. (c) Arrangement for generating several indistinguishable photons which would be detected either from the side port or the end. Here, one would pump the medium via the $v=1$ vibrational level of the electronic excited state. (d) This graphic illustrates how a right-angle geometry detection allows resonant excitation and detection of photons on the 00ZPL.

architecture presented here offers great promise for coupling many individual emitters that have been carefully selected along a one-dimensional bus.

Figure 5(a) plots an excitation spectrum of about 5000 molecules recorded in transmission, displaying a large spectral inhomogeneity. A powerful feature of the nanoguide geometry is that, contrary to focusing with a high numerical aperture and thus a small depth of focus, a guided photon keeps its coupling efficiency along the whole nanoguide length. Application of local electric fields via micro- and nanoelectrodes [see Fig. 5(b)] combined with side-port spectrospatial mapping will allow one to tune a subset of these molecules into resonance [44]. Similarly, one can remove accidental resonances of molecules along the nanoguide. This strategy will make it possible to achieve long-distance polaritonic coupling $[2,10]$ among a well-defined number of molecules that are addressable both spectrally and spatially. Many of these experiments would greatly benefit from larger $\beta$ factors, which can be pursued in different nanoguide geometries such as photonic crystals $[29,30]$ and slot waveguides $[46,47]$.

The possibility of exciting two or more molecules at the same time also provides an opportunity to generate several indistinguishable photons either in transmission or via the side port [see Fig. 5(c)]. Furthermore, as sketched in Fig. 5(d), one can take advantage of the side port to control the population of the excited state of a single molecule via its 
00ZPL [48] instead of the commonly used broad transition to the $|e, \mathrm{v}=1\rangle$ state. As a result, one could gate a singlemolecule transistor [49] with a single photon or only a few, enabling basic nonlinear quantum-optical studies [7,50].

We acknowledge financial support from the European Union (ERC Advanced Grant SINGLEION), the Max Planck Society, and the Alexander von Humboldt Foundation. We also thank the Fiber Technology Development and Service Unit at MPL for providing the glass capillaries and Patricia Schrehardt for cutting and polishing them. S. F. and P. T. contributed equally to this work.

[1] D. G. Angelakis, M. Huo, E. Kyoseva, and L. C. Kwek, Phys. Rev. Lett. 106, 153601 (2011).

[2] A. D. Greentree, C. Tahan, J.H. Cole, and L.C.L. Hollenberg, Nat. Phys. 2, 856 (2006).

[3] D. Gerace, H. E. Türeci, A. Imamoglu, V. Giovannetti, and R. Fazio, Nat. Phys. 5, 281 (2009).

[4] D. E. Chang, V. Gritsev, G. Morigi, V. Vuletic, M. D. Lukin, and E. A. Demler, Nat. Phys. 4, 884 (2008).

[5] I. Carusotto, D. Gerace, H. E. Tureci, S. De Liberato, C. Ciuti, and A. Imamoglu, Phys. Rev. Lett. 103, 033601 (2009).

[6] H. J. Kimble, Nature (London) 453, 1023 (2008).

[7] D. E. Chang, V. Vuletic, and M. D. Lukin, Nat. Photonics 8, 685 (2014).

[8] J.-M. Raimond and S. Haroche, Exploring the Quantum (Oxford University Press, Oxford, England, 2006).

[9] A. Imamoglu, H. Schmidt, G. Woods, and M. Deutsch, Phys. Rev. Lett. 79, 1467 (1997).

[10] M. J. Hartmann, F. G. S. L. Branadao, and M. B. Plenio, Nat. Phys. 2, 849 (2006).

[11] G. Zumofen, N. M. Mojarad, V. Sandoghdar, and M. Agio, Phys. Rev. Lett. 101, 180404 (2008).

[12] I. Gerhardt, G. Wrigge, P. Bushev, G. Zumofen, M. Agio, R. Pfab, and V. Sandoghdar, Phys. Rev. Lett. 98, 033601 (2007).

[13] A. N. Vamivakas, M. Atatüre, J. Dreiser, S. T. Yilmaz, A. Badolato, A. K. Swan, B. B. Goldberg, A. Imamoğlu, and M. S. Ünlü, Nano Lett. 7, 2892 (2007).

[14] G. Wrigge, I. Gerhardt, J. Hwang, G. Zumofen, and V. Sandoghdar, Nat. Phys. 4, 60 (2008).

[15] M. K. Tey, Z. Chen, S. A. Aljunid, B. Chng, F. Huber, G. Maslennikov, and C. Kurtsiefer, Nat. Phys. 4, 924 (2008).

[16] M. Pototschnig, Y. Chassagneux, J. Hwang, G. Zumofen, A. Renn, and V. Sandoghdar, Phys. Rev. Lett. 107, 063001 (2011).

[17] E. W. Streed, A. Jechow, B. G. Norton, and D. Kielpinski, Nat. Commun. 3, 933 (2012).

[18] N. M. Mojarad, V. Sandoghdar, and M. Agio, J. Opt. Soc. Am. B 25, 651 (2008).

[19] M. Sondermann, R. Maiwald, H. Konermann, N. Lindlein, U. Peschel, and G. Leuchs, Appl. Phys. B 89, 489 (2007).

[20] K. G. Lee, X. W. Chen, H. Eghlidi, P. Kukura, R. Lettow, A. Renn, V. Sandoghdar, and S. Götzinger, Nat. Photonics 5, 166 (2011).

[21] D. E. Chang, A. S. Sørensen, P. R. Hemmer, and M. D. Lukin, Phys. Rev. Lett. 97, 053002 (2006).

[22] I. Friedler, C. Sauvan, J. P. Hugonin, P. Lalanne, J. Claudon, and J. M. Gérard, Opt. Express 17, 2095 (2009).
[23] J. T. Shen and S. Fan, Opt. Lett. 30, 2001 (2005).

[24] J. Hwang and E. A. Hinds, New J. Phys. 13, 085009 (2011).

[25] V. I. Balykin, K. Hakuta, F. L. Kien, J. Q. Liang, and M. Morinaga, Phys. Rev. A 70, 011401(R) (2004).

[26] E. Vetsch, D. Reitz, G. Sagué, R. Schmidt, S. T. Dawkins, and A. Rauschenbeutel, Phys. Rev. Lett. 104, 203603 (2010).

[27] R. Yalla, F. Le Kien, M. Morinaga, and K. Hakuta, Phys. Rev. Lett. 109, 063602 (2012).

[28] A. V. Akimov, A. Mukherjee, C. L. Yu, D. E. Chang, A. S. Zibrov, P. R. Hemmer, H. Park, and M. D. Lukin, Nature (London) 450, 402 (2007).

[29] T. Lund-Hansen, S. Stobbe, B. Julsgaard, H. Thyrrestrup, T. Sünner, M. Kamp, A. Forchel, and P. Lodahl, Phys. Rev. Lett. 101, 113903 (2008).

[30] A. Goban et al., Nat. Commun. 5, 3808 (2014).

[31] O. Astafiev, A. M. Zagoskin, A. A. Abdumalikov, Yu. A. Pashkin, T. Yamamoto, K. Inomata, Y. Nakamura, and J. S. Tsai, Science 327, 840 (2010).

[32] S. Ghosh, J. E. Sharping, D. G. Ouzounov, and A. L. Gaeta, Phys. Rev. Lett. 94, 093902 (2005).

[33] M. Bajcsy, S. Hofferberth, V. Balic, T. Peyronel, M. Hafezi, A. Zibrov, V. Vuletic, and M. Lukin, Phys. Rev. Lett. 102, 203902 (2009).

[34] W. E. Moerner and L. Kador, Phys. Rev. Lett. 62, 2535 (1989).

[35] F. Jelezko, P. Tamarat, B. Lounis, and M. Orrit, J. Phys. Chem. 100, 13892 (1996).

[36] D. E. Chang, A. S. Sørensen, E. Demler, and M. D. Lukin, Nat. Phys. 3, 807 (2007).

[37] H. Haakh, S. Faez, and V. Sandoghdar (to be published).

[38] See Supplemental Material at http://link.aps.org/ supplemental/10.1103/PhysRevLett.113.213601 for an intuitive derivation of the relationship between the extinction dip and $\beta$, as well as the distribution of the resonance linewidths in the nanocapillary.

[39] T. Basche, W. E. Moerner, M. Orrit, and U. Wild, Single Molecule Spectroscopy (John Wiley \& Sons, New York, 1999).

[40] H. Walther, in Advances in Atomic, Molecular, and Optical Physics, Vol. 51, edited by H. H. Stroke (Elsevier, New York, 2005), p. 239.

[41] S. Weisenburger, B. Jing, D. Hänni, L. Reymond, B. Schuler, A. Renn, and V. Sandoghdar, ChemPhysChem 15, 763 (2014).

[42] A. Gonzalez-Tudela, D. Martin-Cano, E. Moreno, L. Martin-Moreno, C. Tejedor, and F. J. Garcia-Vidal, Phys. Rev. Lett. 106, 020501 (2011).

[43] C. Hettich, C. Schmitt, J. Zitzmann, S. Kühn, I. Gerhardt, and V. Sandoghdar, Science 298, 385 (2002).

[44] Y. L. A. Rezus, S. G. Walt, R. Lettow, A. Renn, G. Zumofen, S. Götzinger, and V. Sandoghdar, Phys. Rev. Lett. 108, 093601 (2012).

[45] A. F. van Loo, A. Fedorov, K. Lalumiere, B. C. Sanders, A. Blais, and A. Wallraff, Science 342, 1494 (2013).

[46] Q. Quan, I. Bulu, and M. Lončar, Phys. Rev. A 80, 011810 (R) (2009).

[47] Y. C. Jun, R. M. Briggs, H. A. Atwater, and M. L. Brongersma, Opt. Express 17, 7479 (2009).

[48] I. Gerhardt, G. Wrigge, G. Zumofen, J. Hwang, A. Renn, and V. Sandoghdar, Phys. Rev. A 79, 011402(R) (2009).

[49] J. Hwang, M. Pototschnig, R. Lettow, G. Zumofen, A. Renn, S. Götzinger, and V. Sandoghdar, Nature (London) 460, 76 (2009).

[50] J.-T. Shen and S. Fan, Phys. Rev. Lett. 98, 153003 (2007). 